\title{
AN X-RAY TECHNIQUE FOR EVALUATING THE STRUCTURE OF FILMS FOR DEVICE APPLICATIONS
}

\author{
R. N. CLARKE† and IAN N. COURT \\ Physics Division, The Polytechnic, Sunderland, SR1 3SD, U.K.
}

(Received June 26, 1977)

\begin{abstract}
Thin films are composed of many crystallites. Where such films are used in the construction of devices it is frequently necessary to grow them with a preferred orientation. A quick, non destructive method to measure the degree of orientation could provide a useful guide to device performance. We have investigated a technique to obtain this information using the Philips P.W. 1050/25 diffractometer. The method depends on being able to tilt the film out of the diffracting plane of the diffractometer and can be used for films in which all crystallites are orientated to better than $\pm 25^{\circ}$, of the preferred direction. This limit is generally acceptable since greater misorientation will normally produce a poor device. The axial beam divergence of the diffractometer is shown to limit the accuracy of the technique for very highly orientated films. Experimental verification of the method is described.
\end{abstract}

\section{INTRODUCTION}

Many materials when evaporated or sputtered grow on a substrate in polycrystalline form. Under certain circumstances, which depend on the nature and temperature of the substrate during growth, the individual crystallites show a preferred orientation. In a number of device applications, microwave acoustic transducers for example, the existence of these preferred directions is critical to the successful operation of the device and a measure of the degree of alignment of the crystallites is often a useful indicator of device performance.

The determination of preferred orientations or texture is a problem that frequently has to be solved in the study of cold worked metals. Preferred orientations in metals are of importance since their existence can often have a very marked effect on the macroscopic properties of the material. Since the effects are usually undesirable, it is of interest to be able to make a map or pole figure of the statistical distribution of reciprocal lattice vectors of a given set of $(h k l)$ planes within the sample so that any texture will be found.

The most accurate methods for determining pole figures are derived from the $\mathrm{X}$-ray diffractometer methods of Schulz ${ }^{1,2}$ and sophisticated computer controlled X-ray spectrometers now exist which give the complete pole figure for a material.

$\dagger$ Now at Applied Research Unit, W \& T Avery, Walsall, West Midlands.
With thin films used in device construction, it is not usually of interest to see if any texture exists, but rather to know about the orientation of one specific plane. The manner of growth of a typical film is shown in Figure 1. The films are polycrystalline because they grow from a large number of nucleation centres. If the preferred direction of growth is with a particular $\left(h_{1} k_{1} l_{1}\right)$ plane parallel to the substrate, then we can characterise a crystallite growing in this preferred direction by a unit vector $\underline{N}_{0}$ which is parallel to the reciprocal lattice vector of the $\left(h_{1} k_{1}\right.$ $\left.l_{1}\right)$ plane and normal to the substrate. Similarly we can characterise each crystallite in the film by a unit vector normal to its $\left(h_{1} k_{1} l_{1}\right)$ plane. Thus the unit vector $\underline{N}_{j}$ in Figure 1 represents the jth crystallite which is orientated so that $\underline{N}_{j} \cdot \underline{N}_{0}$ is the cosine of the angle it makes with the preferred direction. We

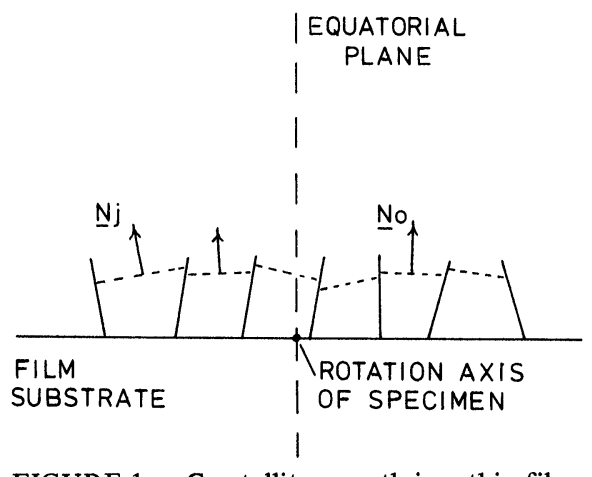

FIGURE 1 Crystallite growth in a thin film. 


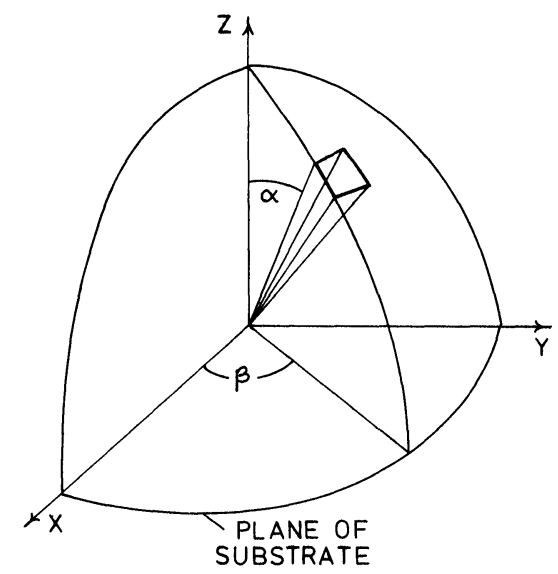

FIGURE 2 Co-ordinate system for describing orientation of crystallites.

can write a distribution function for these unit vectors. Referring to the co-ordinate system in Figure 2 , the distribution function $N(\alpha, \beta) \mathrm{d} \Omega$ is the number of crystallites orientated within an elemental solid angle $d \Omega$ of the direction $(\alpha, \beta)$. Good device performance requires highly orientated films with $N(\alpha, \beta)$ negligibly small for values of the angle $\alpha$ greater than about ten degrees. We have investigated a commonly available X-ray diffractometer, the Philips P.W. $1050 / 25$, to see if it can be used to determine such restricted forms of $N(\alpha, \beta)$. Van Someren and Sprenkle $^{3}$ have also investigated the possibility of modification of this diffractometer to obtain pole figures. However, they were concerned with a very elaborate modification which would enable them to obtain complete pole figures with cylindrical specimens. In their work they did not take account of the axial divergence of the X-ray beam, a factor which we have found to be important in investigating very highly orientated films.

The simple technique was developed for a study of zinc oxide films ${ }^{4}$ but is suitable for any film in which the lateral extent of the crystallites is sufficiently large $(>10 \mathrm{~nm})$ and for films which are not too severely stressed so that Bragg scattered radiation from the films has a linewidth of about one degree or less. The technique is described in section 2 . The orientation texture of crystals can also be found by photographic records of X-ray scattering, and in section 3 we compare the results of the technique with the results of a microdensitometer study of films produced in a forward reflection camera. In section 4 we investigate the limitations of the method.

\section{X-RAY DIFFRACTOMETER TECHNIQUE}

The Philips PW 1050/25 diffractometer attachment was mounted on the PW 1130/100 power unit. A simple holder was designed for the substrate on which the films were grown which would fit into the diffractometer sample chamber and would position the film so that it coincided with the axis of the diffractometer so that the Bragg reflections from the $\left(h_{1} k_{1} l_{1}\right)$ planes characterised by vectors parallel to $\underline{N}_{0}$ could be detected. The holder was made of aluminium and masked with plastic tape so that no Bragg reflections from the aluminium could be observed. The effect of the holder was to produce an insignificant increase in the background radiation. The special feature of the holder was that it enabled the film to be tilted through and angle $\alpha$ about the axis in the diffracting plane at right angles to the diffractometer axis. See

Figure 3. The tilt angle could be set with an accuracy of one degree. As can be seen by inspection of Figure 1, the effect of tilting the film is to bring the $\left(h_{1} k_{1} l_{1}\right)$ planes of misaligned crystallites into the configuration where Bragg reflections from them will be detected. The precise design of the holder is not important and is determined by the size and shape of the substrate being used.

Measurements were made on gold films which were grown preferentially orientated, so that the (111) planes were all nearly parallel to the substrate. The $\mathrm{X}$-ray source was nickel filtered copper $\mathrm{K} \alpha$ radiation. The source has an axial length of $10 \mathrm{~mm}$ and the $\mathrm{X}$-rays have an axial divergence of $4.6^{\circ}$ and diverge $1^{\circ}$ in the equatorial plane. The divergence of the beam is

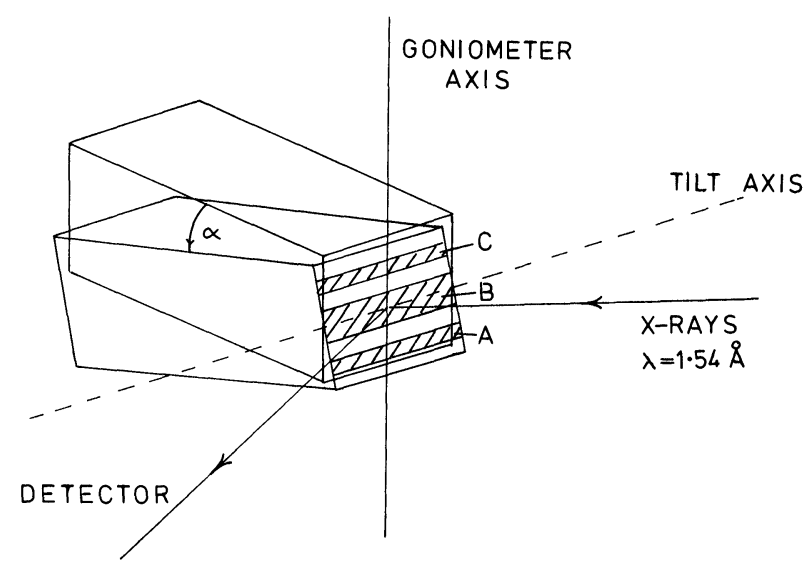

FIGURE 3 Orientation of sample in the diffractometer. The shaded areas A, B, and C are regions of the film which lie above, in, and below the diffracting plane respectively when the specimen is tilted through an angle $\alpha$. 


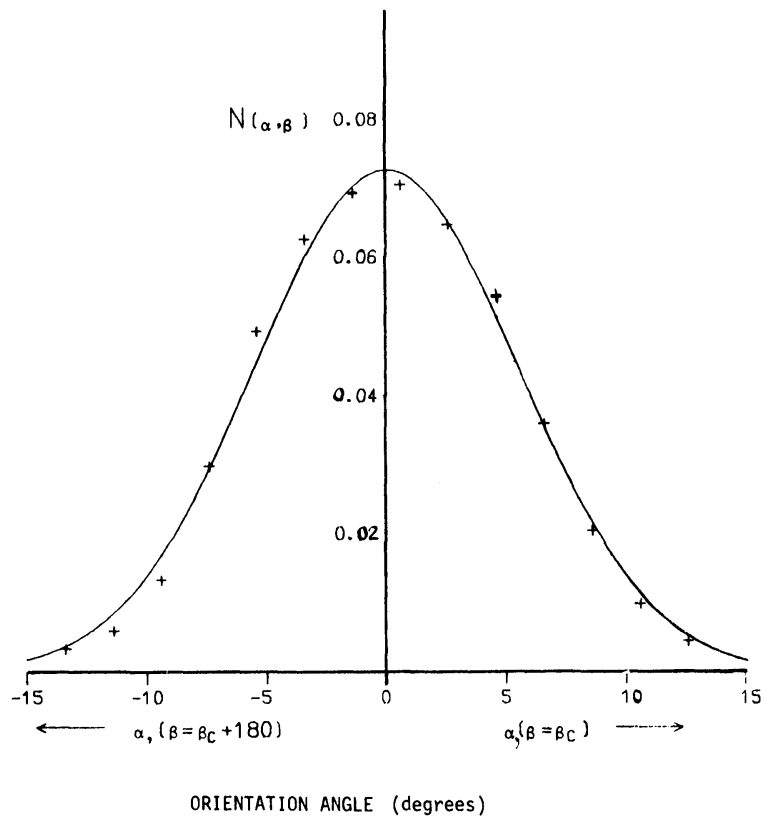

FIGURE 4 Distribution of crystallite orientations as a function of angle for a gold film. S. Dev. $=5.5$

such that the $6 \mathrm{~mm}$ square gold films were uniformly irradiated.

The Bragg angle for the gold (111) planes was $19.09^{\circ}$ and the linewidth of the scattered beam was found to be $0.3^{\circ}$. The Bragg scattered radiation was detected by a proportional counter. The scattered intensity required for any particular position of the substrate normal is the integrated intensity of the reflection (see section 4) above the background intensity. This quantity was measured for each fixed $(\alpha, \beta)$ by scanning the diffractometer over the interval between two background positions $2 \theta_{1}$ and $2 \theta_{2}$ $\left(2 \theta_{1}<2 \theta_{\mathrm{B}}<2 \theta_{2}\right)$ and then subtracting the background count from the integrated counts.

The integrated count for a given $(\alpha, \beta)$ is a measure of the proportion of crystallites at that angle. The results for a $1 \mu \mathrm{m}$ thick gold film are shown in Figure 4. The solid curve is a Gaussian whose standard deviation gave a best-fit to the data. The Gaussian has been normalised so that

$$
\iint N(\alpha, \beta) d \Omega=1
$$

area of hemisphere

Figure 4 shows part of a diameter of a pole figure.

The symmetry of the curve for positive and negative values of $\alpha$ indicates the films have a fibre texture confirming the work of Vook and Witt ${ }^{5}$ who showed the presence of texture in gold films by a study of the relative intensities of Bragg reflections from a number of different crystal planes. The presence of fibre symmetry together with our need of highly orientated rather than randomly orientated films has led us to characterise the films by the standard deviation of the Gaussian rather than the more usual orientation index. ${ }^{6}$

\section{POLAR PLOTS}

Polar photographs of X-ray scattering from randomly orientated films show the familiar circles of powder diffraction. For films with partial orientation these rings are reduced to arcs. It is easily shown that there is a geometrical relationship between the orientation of a crystal plane and the position on the arc of the Bragg scattered X-rays. With a film positioned so that the incident X-ray beam makes the Bragg angle, $\theta_{\mathrm{B}}$, with the plane of the substrate, crystallites orientated at an angle $\alpha$ to the normal produce an image on the film at an angle $\phi$ from the plane of incidence (see Figure 5) where

$$
\cos \alpha=\cos ^{2} \theta_{\mathrm{B}} \cos \phi+\sin ^{2} \theta_{\mathrm{B}}
$$

If the characteristics of the photographic film are known and the material can be orientated so that no
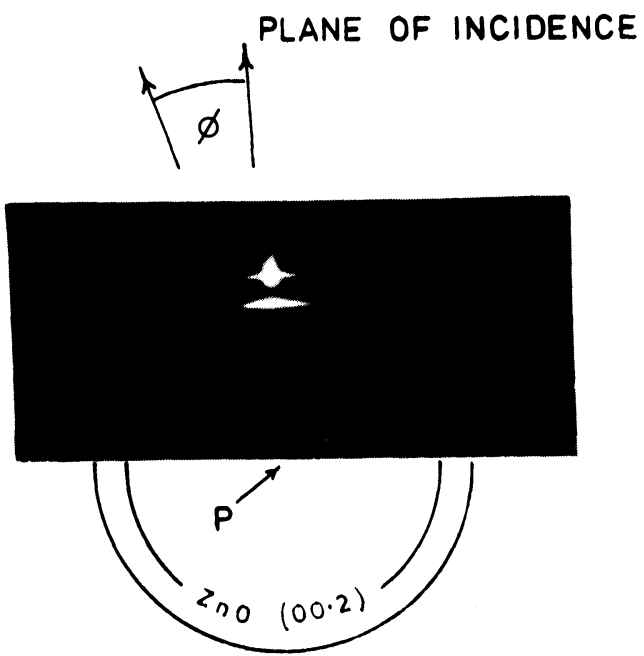

FIGURE, 5 Polar plot produced in a forward reflection camera of X-rays scattered by a $\mathrm{ZnO}$ film. The plane containing the incident $\mathrm{X}$-ray beam and the beam diffracted from those crystal planes which are parallel to the substrate we have called the plane of incidence. The Bragg reflections from the $\mathrm{ZnO}(00.2)$ planes appear as a short arc showing the crystallites to be moderately well orientated. 


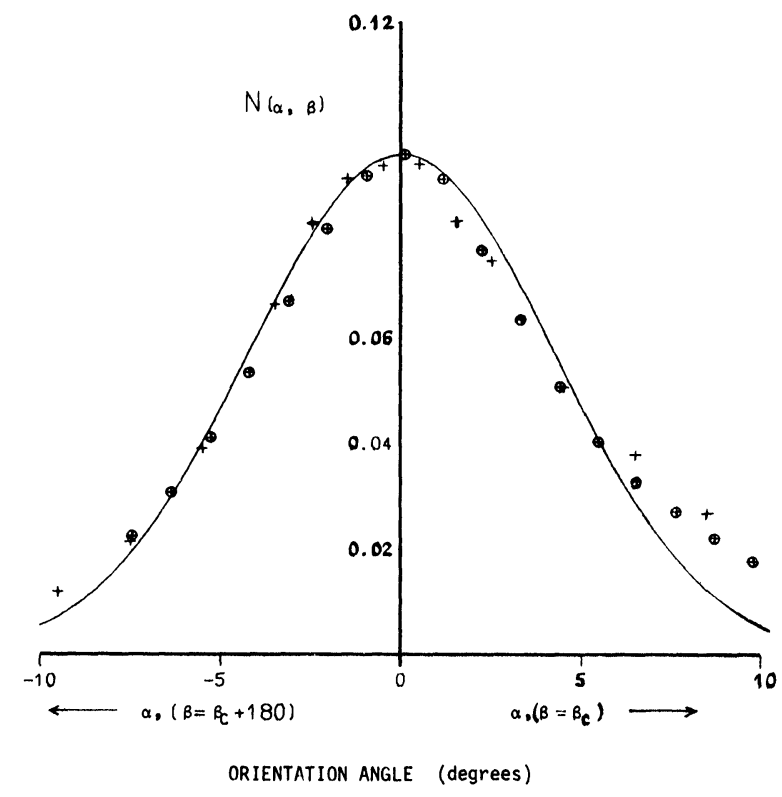

FIGURE 6 Distribution of crystallite orientations as a function of angle for a $\mathrm{ZnO}$ film. S. Dev. $=4.2^{\circ} . \oplus \mathrm{X}$-ray-film data, + X-ray diffractometer data.

corrections for absorption are necessary, the relative density of the darkened film around the arc is a measure of the degree of orientation of the crystallites.

Figure 5 shows the polar pattern of diffracted nickel filtered copper $\mathrm{K} \alpha$ radiation for a zinc oxide film, taken in a Philips PW 1008 camera. The X-ray pattern is for the Bragg reflections at $17.2^{\circ}$ from the (00.2) planes of a $1.25 \mu \mathrm{m}$ thick film. The pattern was recorded on Ilford Industrial $\mathrm{G}$ film with an exposure of two hours.

The density of this film was measured on a Joyce Loebl CS3 Microdensitometer. This instrument has a resolution of $5 \mu \mathrm{m}$ square. The microdensitometer was programmed to make a raster scan of the film and to record the integrated intensity of each line and the location of the peak intensity for each $\mathrm{X}$ scan. ${ }^{7}$ In this way an accurate profile of the intensity around the arc was obtained. The centre of the ring system (point P Figure 5) was determined by inspection and Eq. (1) used to identify the orientation of the crystal plane which produced the density at each point along the arc on the film. The relative density of the film is proportional to the number of crystal planes of the orientation which satisfy the Bragg condition. A plot of density vs crystallite orientation is shown in Figure 6.

On the same curve the results of the diffractometer measurements are shown. The film data has been $\oplus \quad X$-RAY FILM DATA

$+\quad X$-RAY DIFFRACTOMETER DATA

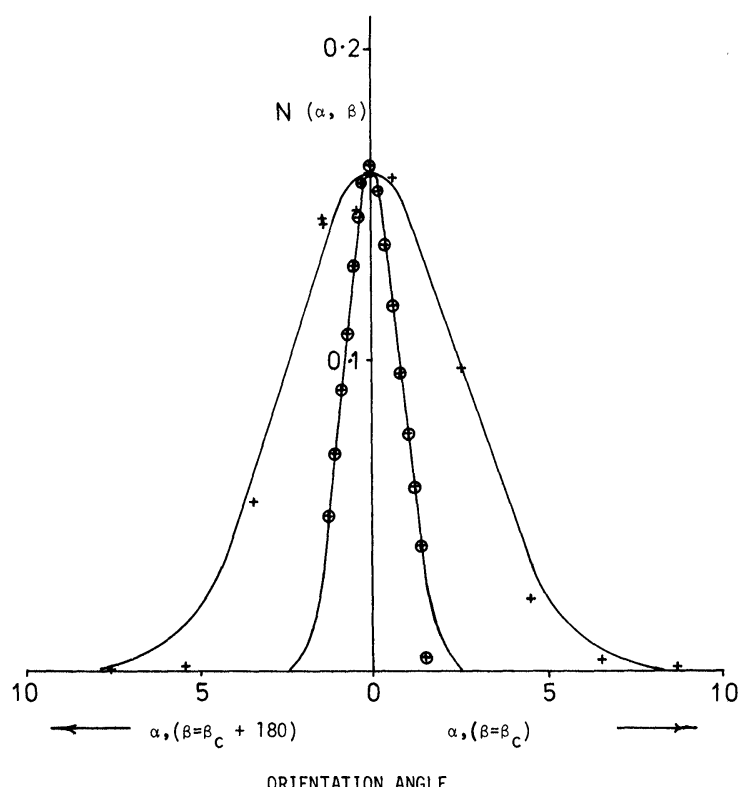

FIGURE 7 Distribution of crystallite orientation as a function of angle for a highly orientated gold film.

normalised so that the peak intensity is the same as for the diffractometer data. The solid curve is a bestfit Gaussian and it is evident that the two techniques lead to similar results. Measurement on other films with $\sigma>4^{\circ}$ gave similar results. However, for very highly orientated films, $\sigma<3^{\circ}$, no such agreement was found. Figure 7 shows the two sets of data for a very highly orientated gold film.

\section{SOURCES OF ERROR}

The disagreement between the results obtained from the two methods of measurement on very highly orientated films could not be accounted for by errors in measurement. However, there are a number of effects which may cause the measurements on the crystallite distribution to be in error. We have isolated four.

Firstly, the width of the measured $N(\alpha, \beta)$ curve is not entirely due to the distribution of crystallites about the normal. It is also affected by the linewidth of the Bragg scattered line irrespective of whether this is inherent in the instrument or caused by the crystallite size or stress in the film. The linewidth has been measured for all our films. Typical measure- 
ments show the linewidth $B_{1 / 2}$ to be $0.30^{\circ}$. This broadens the $N(\alpha, \beta)$ curve by $0.01^{\circ 8}$ which is negligible compared with our measured values which were all greater than one degree.

Secondly, as the sample is tilted, its effective cross sectional area is reduced and it intercepts less of the $\mathrm{X}$-ray beam. Moreover the path of the X-ray beams through the film is increased, thus increasing the loss of intensity because of absorption in the film. A straightforward calculation has been made to see how these geometrical factors will affect the results. For gold films, taking $\mu / \rho=213 @ 0.154 \mathrm{~nm}$, the reduction in intensity is less than ten per cent for $|\alpha|<25^{\circ}$. For thinner films and/or ones made of lighter elements, still larger values of $\alpha$ could be tolerated.

Thirdly, tilting the film moves it out of the diffracting plane. The film can be divided up into three zones labelled A, B and C in Figure 3, which lie above, in, and below the diffracting plane respectively. Bragg scattering from the $\left(h_{1} k_{1} l_{1}\right)$ planes of crystallites in the zone $B$ will be detected at the normal Bragg angle setting of the diffractometer. The equatorial plane beam divergence is sufficiently large that Bragg reflections from the $\left(h_{1} k_{1} l_{1}\right)$ planes in zone A occur but they are observed at an apparently larger 'Bragg' angle. Similarly reflections from crystallites in zone $\mathrm{C}$ appear at a smaller 'Bragg' angle. This problem has been investigated by Chernock and Beck ${ }^{9}$ and is overcome by using the integrated intensities as the measure of the number of crystal planes of a given orientation.

Fourthly, in pole figure work, the X-ray beam is highly collimated; the axial beam divergence being limited by special slits. In the PW 1050/25 the axial beam divergence, $\Delta$, is limited by Soller slits to $\Delta=2.30^{\circ}$

If $\delta$ is the angle which a given $\mathrm{X}$-ray makes with the equatorial plane, then the intensity of the beam which emerges from between each pair of plates of the Soller slits has a maximum value at $\delta=0$ and falls to zero for $\delta=\Delta$ where $\Delta$ is determined by the geometry of Sollet slits. X-rays scattered out of the equatorial plane are prevented from reaching the detector by another set of Soller slits. Again the slits do not achieve perfect collimation and the diffracted beam profile is similar to the incident beam profile. This axial divergence of the incident and detected $X$-rays results in the detection of diffraction from crystal planes orientated at angles $\alpha^{1}$ to the substrate normal where $\alpha^{1}$ may be larger or smaller than the angle of tilt, $\alpha_{0}$, of the substrate holder.

Consider the situation where the incident X-ray AO is in the equatorial plane. (Figure 8). In addition to

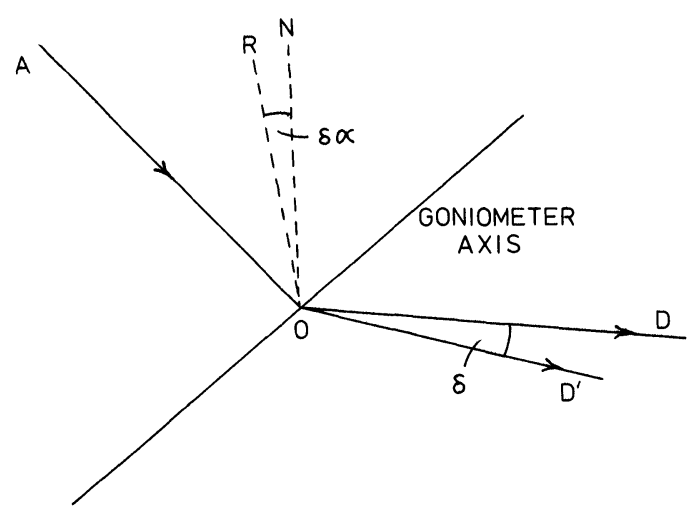

FIGURE 8 Scattering of X-ray beams from nearly parallel crystal planes.

the diffracted beam OD which also lies in an equatorial plane, a diffracted beam $\mathrm{OD}^{\prime}$ making an angle $\delta \alpha=\alpha^{1}-\alpha_{0}$ to the equatorial plane will also be detected. If the normals to these diffracting crystal planes are $\mathrm{ON}$ and $\mathrm{OR}$ respectively then the angle between these normals can be shown to be

$$
\cos (\delta \alpha)=1+\frac{\cos \delta-1}{4 \sin ^{2} \theta_{B}}
$$

Thus for $-\Delta<\delta<\Delta$, the detector will respond to scattering from crystallites orientated such that $\alpha^{1}>\alpha_{0}+3.5^{\circ}$ and $\alpha^{1}<\alpha_{0}-3.5^{\circ}$. For randomly orientated crystallites, the detected diffracted beam has maximum intensity for reflections from crystal planes making an angle $\alpha_{0}$ with the substrate normal. The detected intensity falls to zero for reflections from crystal planes making angles $\alpha^{1}>\alpha_{0}+3.5^{\circ}$ and

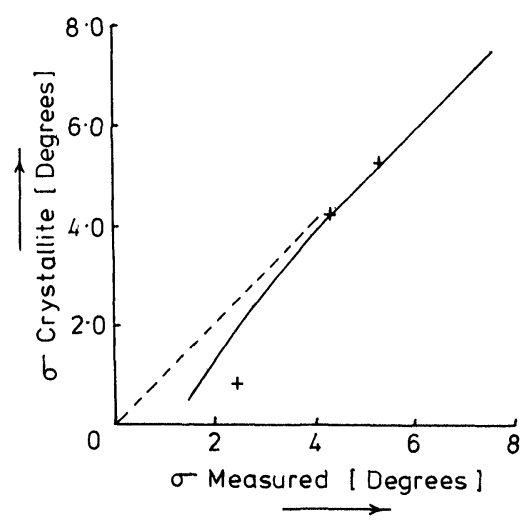

FIGURE 9 Apparent broadening of the crystallite distribution caused by the effects of axial beam divergence. The theoretical curve based on the assumption that the $\mathrm{X}$-ray beam profile is triangular is shown by the solid line. 
$\alpha^{1}<\alpha_{0}-3.5^{\circ}$. The axial divergence will have no effect on the intensity which will remain independent of the angle of tilt. However, it will cause the observed crystallite distribution of orientated films $N(\alpha, \beta)$ to be broader than the true crystallite distribution. If we assume that the beam intensity profiles are triangular then for a known distribution of crystallites $N(\alpha, \beta)$ we can calculate the expected measured distribution. The results are shown by the solid line in Figure 9. This broadening is confirmed by a comparison with the photographic data.

\section{CONCLUSION}

The Philips P.W. 1050/25 X-ray diffractometer can be simply modified to make it suitable for determining limited pole figures for films whose spread of orientations about the preferred direction is not too large. Samples up to $28 \mathrm{~mm}$. long can be examined. Since the X-rays completely penetrate the film and illuminate the whole area, the partial pole figures obtained are average results for the whole film.

For well orientated films with $|\alpha|<25^{\circ}$, the technique is more sensitive than that employing measurement of the relative intensities of Bragg reflections from different crystal planes. Interpretation of the diffractometer results is much easier than interpretation of the film density data obtained in the forward reflection camera. However, the axial beam divergence limits its usefulness with very highly orientated films $\sigma<2^{\circ}$. It is therefore of no use for examining epitaxially grown films.

The main advantage of the technique is that it is cheap, non-destructive and enables a quick check to be made on the quality of the film, giving useful information for device applications.

\section{ACKNOWLEDGEMENTS}

We wish to thank Mike Hern for his assistance in the X-ray diffraction work, Gordon Percival for general assistance in the construction of equipment and photographic work, and Joyce Loebl Co. Ltd., for making their microdensitometer together with appropriate software available to us.

\section{REFERENCES}

1. L. G. Schulz, "A Direct Method of Determining Preferred Orientation of a Flat Reflection Sample Using a Geiger Counter X-ray Spectrometer", J. App. Phys., 20, pp. $1030-3$, November 1949.

2. L. G. Schulz, "Determination of Preferred Orientation in Flat Transmission Samples Using a Geiger Counter X-ray Spectrometer", J. App. Phys., 20, pp. 1033 -8, November 1949.

3. L. Van Someran and E. Sprenkle, "Goniometer for Partial Pole Figure Determination on Cylindrical Specimens", Review of Scientific Instruments, 40, pp. 56-60, January 1969 .

4. R. N. Clarke and Ian N. Court, "The Growth of Polycrystalline Films of Zinc Oxide", presented at the Symposium of the Crystallography group of the Institute of Physics on the Characterisation of Polycrystalline Materials by X-ray Diffraction, London, 1976.

5. R. W. Vook and F. Witt, "Structure and Annealing Behaviour of Metal Films Deposited on Substrates near $80^{\circ} \mathrm{K}$, II Gold Films on Glass", J. Vac. Sci. Tech, 2, pp. 243-9, 1965.

6. ASTM Designation E 81.63 "Standard method for preparing Quantitative Pole Figures for Metals", pp. $151-167,1963$.

7. J. Lewis, "Peak Spotting Programme, Peaks", MDM3CS/NOVA, Joyce Loebl Co. Ltd., Gateshead, 1976.

8. A. J. C. Wilson, "Mathematical Theory of X-ray Powder Diffractometry", Philips Technical Library, Eindhoven, The Netherlands, 1963.

9. W. P. Chernock and P. A. Beck, "Analysis of Certain Errors in the X-ray Reflection Method for the Quantitative Determination of Preferred Orientations", J. App. Phys., 23, pp. 341-5, March 1952. 

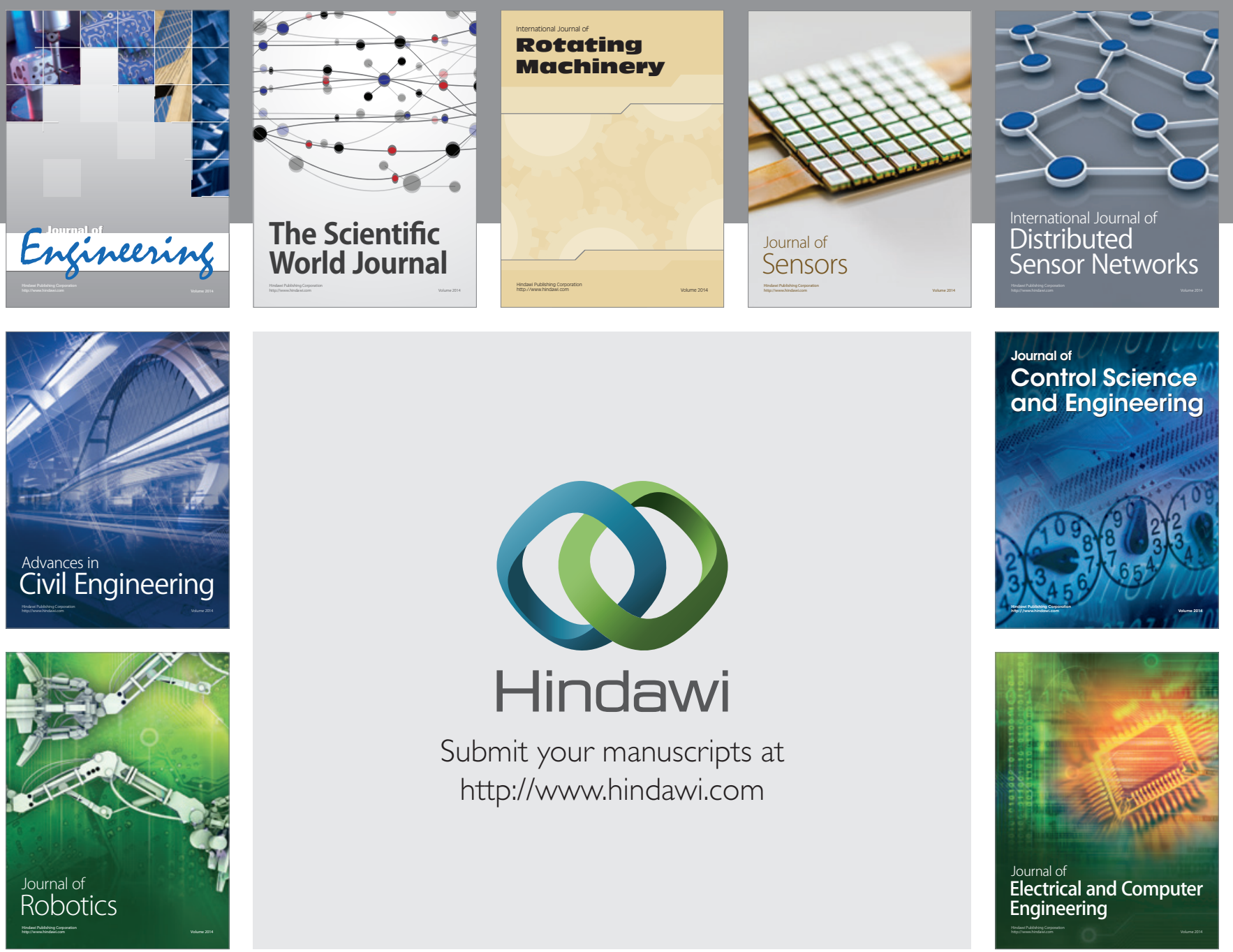

Submit your manuscripts at

http://www.hindawi.com
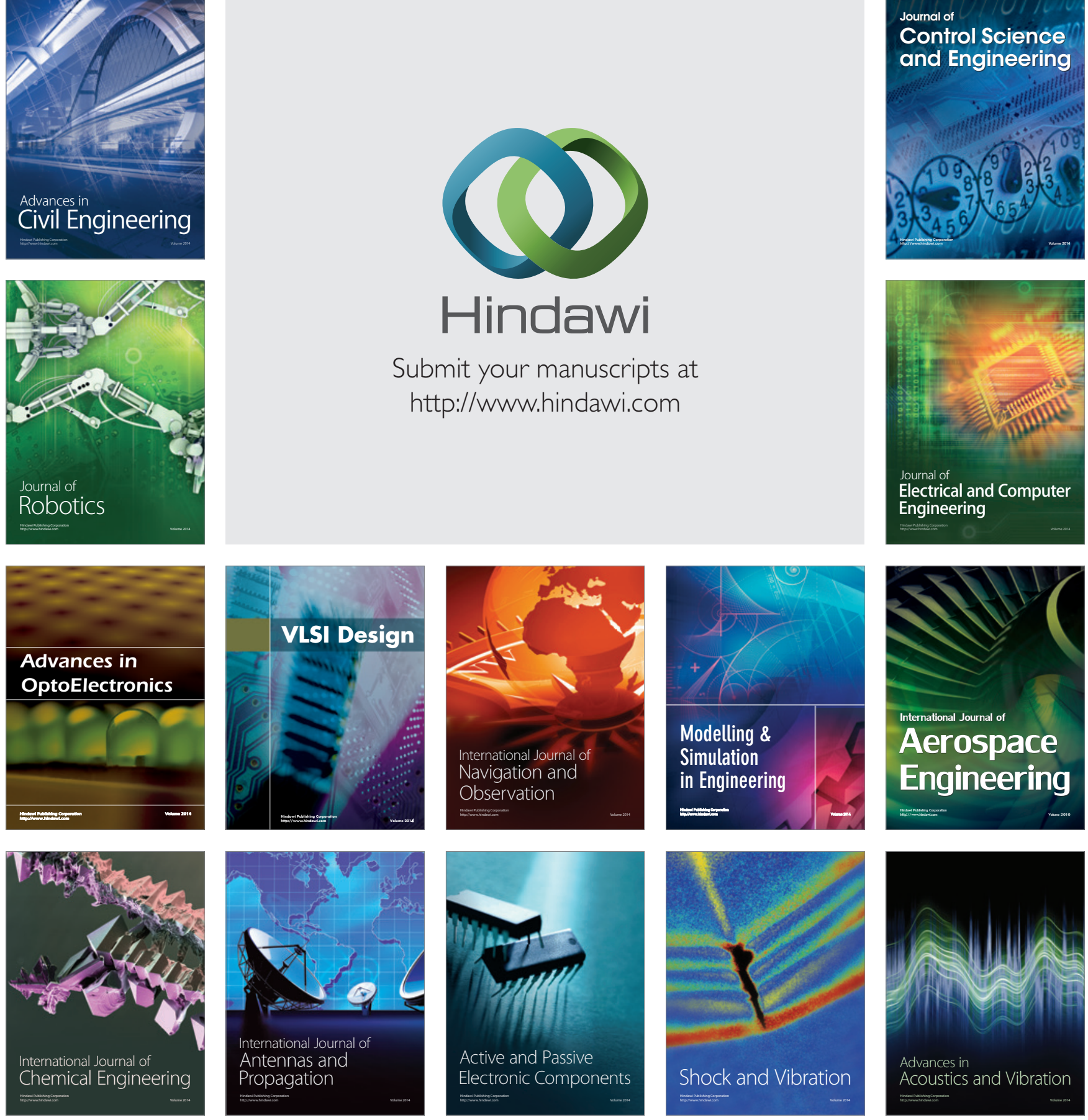